\title{
Using mobile media creation to structure museum interpretation with professional vision
}

\author{
Angeles Muñoz Civantos ${ }^{1} \cdot$ Michael Brown ${ }^{1} \cdot \operatorname{Tim}_{\text {Coughlan }}^{2} \cdot$ \\ Shaaron Ainsworth ${ }^{1} \cdot$ Katharina Lorenz $^{1}$
}

Received: 7 April 2015/ Accepted: 19 November 2015/Published online: 11 January 2016

(c) The Author(s) 2016. This article is published with open access at Springerlink.com

\begin{abstract}
Mobile technology plays an increasing role in museum and cultural heritage contexts. In most cases, these tools support the relatively passive consumption of expert interpretations, or the unguided generation of content by users. This paper explores the potential for technologies to help museum visitors, encountering unfamiliar objects, to engage with them as a skilled professional interpreter would, through structured mobile experiences that focus on creating multimedia content. We explore this concept in the area of artefact interpretation and specifically how to enact a structured process of interpretation, as would commonly be taught in courses dedicated to the analytical diagnostics of visual evidence, such as Classical Archaeology or Art History. We discuss two field trials of prototype systems through which the structured creation of multimedia forms a basis for learning to interpret historical artefacts conducted in contexts of both formal and informal learning. By describing, implementing, and evaluating this approach, we contribute understanding of a new way to conceptualise active engagement in museum contexts, through the effective use of scaffolding and user generation of multimedia. We identify issues around the properties and flexibilities of multiple media for this purpose, links between provision for procedural and factual learning, and the value of media creation-based structures in improving the skills and confidence to interpret.
\end{abstract}

Keywords E-learning - Entertainment - Interaction design $\cdot$ User studies $\cdot$ Scaffolding

Michael Brown

michael.brown@nottingham.ac.uk

1 University of Nottingham, Nottingham, UK

2 Open University, Milton Keynes, UK

\section{Introduction}

Museum, attraction, and cultural heritage visiting has become an important context of research for HCI, UbiComp and Educational Technology. Work in this area has developed and evaluated various forms of guides, uses of locative media, and enhancements to the visitor experience. Visiting attractions such as museums is a commonplace form of learning. This can occur formally as part of a field trip, or informally, as a setting in which learning occurs through cultural visiting. Technologies deployed in these areas have been seen to have the capacity to provide information, interpretations, or to help to generate mementos $[5,8,21]$.

This paper focuses on end-user content creation as an active process that can be structured in various ways, in order to guide users through a process of interpretation of an artefact. We intend to create a sweet spot between enabling the user's own interpretative and creative approaches to come forward, and supporting the user to learn and apply a 'professional vision'-the socially organised ways of seeing and understanding [10] that experts use in their processes of interpretation.

It is now well understood that to improve the potential of technology to aid learning, it is important to go beyond the passive provision of information, towards more 'active' learning approaches that engage and develop problem solving, communication, or collaboration skills $[4,13,19$, 21]. However, such approaches are far more common within formal education [6]. In formal settings, learners can be expected to have substantial time allocated to learn topics and to become familiar with technologies, with support from knowledgeable teachers. Additional challenges for museums as they cater for informal learning is that visitors will come with widely differing prior 
knowledge and experience, will have a range of goals for their visit, and are less likely to have the support of a knowledgeable other [6].

One strand of relevant prior work has created technologies that structure the learning of scientific inquiry in both formal and informal learning [4, 17, 24]. This approach allows topics of personal interest to be explored, while providing scaffolding to follow and so learn a standardised scientific procedure. Here we explore whether and how similar techniques can be applied to interpretation of artefacts, by building and evaluating systems that structure the user's interpretation activity according to processes understood by experts, as taught in classical-archaeological and art-historical education.

A second strand of relevant research has highlighted the potential for the creation of photo-stories or media-based comics, both in school science education [22], and as a means to produce engaging mementos of visits to attractions [5]. At the present time, the status of user generation of media in museums as an 'active' learning process is uncertain, with one study suggesting that basic acts of photo taking might be detrimental to recognition and memory of the artefacts studied. However, more detailed activities such as photographing parts of an artefact appear to counter this 'photo-taking-impairment' effect [11]. More generally, the active use of mobile devices can add cognitive demands in a context that is already full of new sensory information. However, such use also provides scope for multiple forms of representation to be interacted with, therefore holding potential to aid knowledge integration and movement between different levels of detail [21].

A review of research that seeks to bridge the gap between formal and informal learning by science students suggests a variety of findings that are relevant: That time spent attending to museum exhibits can be predictive of learning; that time spent and learning can be increased by greater interactivity or capacity for manipulation; that student's attitudes are more positive towards structured exhibits, rather than those lacking structure; and that guidance may be more valuable to learning than exhibit labels [12].

Further exploration is therefore required to consider whether and how media creation with mobile devices-a now commonplace activity in visiting experiences-has value as a means of active engagement that could promote learning. In particular, the research above suggests the potential importance of scaffolding through technological guidance (e.g. systems that prompt visitors to go beyond simple photo taking, and explore the details of an artefact). Here we design and evaluate such a form of scaffolded media creation that guides users towards carrying out the process of interpreting artefacts through the structure of a simplified expert model [27]. Our tool/activity constitutes scaffolding as it gives users enough assistance to learn the process of interpreting artefacts through a structure/expert model that shows the expert way of carrying out the task [27] by breaking the task of interpreting an artefact into smaller tasks, facilitating the understanding of the process. Our aim is that users will therefore be able to organise their interpretive ideas through more specific questions [20], which promotes a deeper understanding and new knowledge [27].

Given the domain, specific elements of scaffolding were developed on the basis of the art-historical method of iconology [16]. Through this, we aimed to help users becoming aware of the process of interpreting an artefact, to then be able to apply the strategy proposed by the expert model in other situations [17], and ultimately, to analyse and interpret an artefact independently.

A final strand of work that we build upon considers how to design technology to support the sharing of a process involving interpretation in the context of cultural visiting. In prior work, 'gifting experiences' to others was found to promote personalisation and increase the social aspects of visiting [8]. As an extension of this, the work described in this paper explores how structures representing an interpretation process could be firstly designed by experts in a field, and then shared with multiple individuals, who then create their own interpretive responses within the given structure. Such an approach could fit with both the value of designing for multiple meanings and interpretations, identified in HCI literature [9, 23], and trends within the museum sector to encourage the opening up of authoritywith interpretation being no longer solely the preserve of curators and experts $[18,25]$. The approach defined here repositions the role of these experts in the museum towards the sharing of procedural knowledge for their individual reuse.

In the following sections, we describe two prototype design and evaluation studies that employ this approach in two different museum settings, and within formal and informal learning situations. Through this, we can evaluate instances of the system across a spectrum of potential users and uses. In both of the studies, we make use of different levels of structure in the activity, not as an experimental methodology but as a qualitative explorative field trial of interactions with the technology, following the tradition of such studies in this domain.

Our contribution in this paper is to outline and evidence a potential approach for more active, guided engagement through technology in museums. This balances the provision of structures through which a procedure for interpretation can be followed, with space for the content of these interpretations to be produced by the individual. In evaluating this concept, we explore how such an approach can 
increase the ability and confidence to interpret objects, and also explore how various types of media creation activities play a role in achieving this active engagement.

\section{Initial study: designing an experience in the context of formal classical-archaeological and art-historical education}

\subsection{Approach to design}

In order to explore the area outlined above, we designed a mobile experience that adopted processes used in formal classical-archaeological and art-historical education and scholarship, as a basis for a new form of museum-based activity. As a first step, a formal learning situation had the advantage of being more familiar and well-defined as a starting point for the cross-disciplinary research team, when compared to informal learning. For example, there were expected learning outcomes and related existing activities from courses that could be discussed and drawn upon when designing the activity. Classical Archaeology, as other areas of culture-historical study, continuously draws upon the analysis of physical artefacts; hence, interpretation skills are a key learning outcome from courses in the subject.

To develop the basic form of the activity (outlined in Table 1), a classical archaeologist and learning scientist worked together to represent structures and knowledge of the interpretation of historical artefacts in a way that was akin to the procedures expected to be learnt during a formal education in classical-archaeological and art-historical analysis and interpretation. Subsequently, a series of three design workshops were held with experts in artefact interpretation, education, curation and technology design to further refine the design. The results of these workshops were informed by Panofsky's three-step model of art-historical analysis and interpretation [16]. A model of the procedure to be learnt was formalised. This model then formed the basis for the design of a structured mobile experience by a team including computer science, $\mathrm{HCI}$ and art-historical expertise. In this context, and inspired by prior work involving media creation in visiting contexts [5, $11,22]$, it was decided to facilitate the construction of multi-modal interpretations through the creation of different media (video, images, audio recordings, and text), which would represent the user's interpretation.

The intent of the research team was that could also be envisaged as a system to potentially replace outcomes that might typically take the form of a written assignment in classical-archaeological and art-historical education. It was also noted for future work that field trips often had very limited outcomes, or limited use of the experience gained after the event, and that this approach could be connected into longer term teaching and learning strategies in a variety of innovative ways.

\subsection{Prototype development}

In order to explore and evaluate the potential of structured interpretation activities that combined professional vision with media creation, two contrasting experiences were designed; a structured activity based on the model of interpretation described above in Table 1, and an

Table 1 The initial structure for the activity designed by experts in classics and learning science

Step 1: Technical description ( $\sim 5 \mathrm{~min})$

Step 1.1 Space, location and subject matter

Step 1.1 Material

Step 1.2 Condition/change

Step 2: Systematic description ( $\sim 15 \mathrm{~min})$

Step 2.1 Individual components of the object

Step 2.2 Relationship of the individual components of the object

Step 3: Iconographic comparison $(\sim 10 \mathrm{~min})$

Step 3.1 Input

Step 3.2

Step 4: Interpretation $(\sim 10 \mathrm{~min})$

Step 4.1
Object basics: Record the space and place of the object and what is represents

Material: Record information about the material

Restoration: Record and describe the parts that are damaged or show modern restoration

Components: Record and describe the individual components. In this case: face and hair, dress, body posture; attributes; female $\mathrm{a} / \mathrm{b}$

Relationships: Record and describe the relationship of the individual components. In this case: the relationship between female $a / b$, incl. also relationship to any attributes or surrounding features

Present a choice of objects used for iconographic comparison with the object focused on-include objects that are particularly relevant, but also include objects which are not relevant; slideshow of objects with brief descriptions

Comparison: Respond on relevance of comparative material

Record information about interpretive judgments such as date of creation and what the object represents 
unstructured activity, which supported the capture of media and text about an object in a basic way, as a means of comparison that would allow us to evaluate the impact of the devised structure on the experience of users.

Both experiences were implemented using the Wordpress platform. This allowed the rapid development of mobile-optimised, web-based structures that could prompt the user in various ways, and capture their responses in multiple media types. These allowed the collection of multimedia in the museum and gallery space, and prompted users to complete tasks in a linear series of stages, through capturing media and answering questions.

\subsubsection{The structured experience}

The structured experience was designed to formalise an artefact agnostic process for interpreting artefacts that would be accessible to beginners, yet give them an appreciation of the professional vision of the expert classical archaeologist. The activity was designed so that users were directed to create media responses to system prompts, following the structure defined in Table 1. Tasks could involve taking photographs of the artefacts, making audio and video recordings, text entry, or a combination of these. Through this approach, the design aimed to encourage active viewing, an active engagement with the artefacts by creating individual media responses, rather than passive viewing of museum objects.

For each prompt, users are expected to provide short text interventions alongside pictures, video or audio, in order to describe and capture particular aspects of the artefact. Multiple-choice questions were also given as part of Sect. 4 , in order to explore the potential of this form of interaction to prompt structured interpretation. At the beginning of each stage, participants were advised to spend

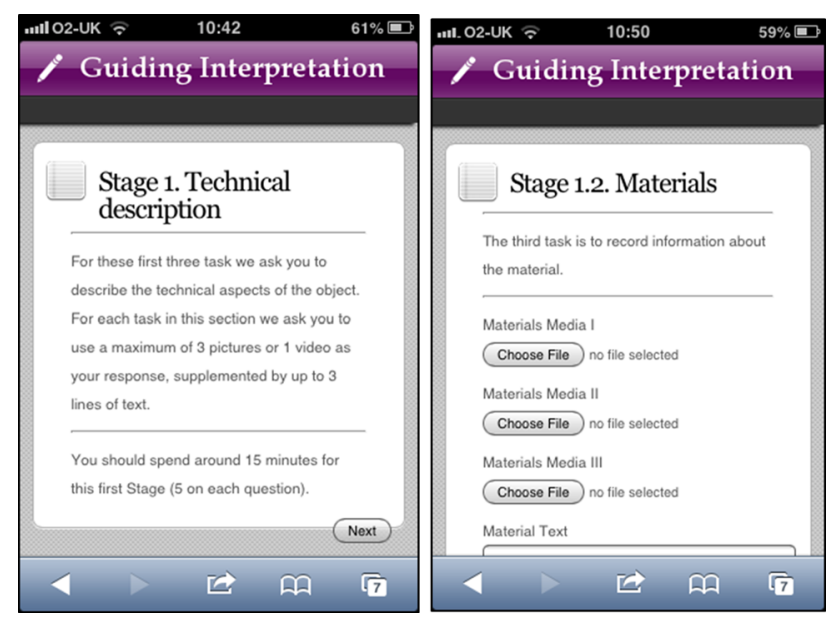

Fig. 1 Example screenshots from the structured activity a set period of time to complete the given tasks, but this timing was not enforced (Fig. 1).

\subsubsection{The unstructured experience}

The unstructured experience allowed participants to share their thoughts and reflections and to capture a range of media: video, text, photographs and audio recordings, via a web-interface. In contrast to the structured experience, it gave no prompts or guidance as to what to focus on or capture. Instead it only provided spaces in which to add each type of media in connection with the relevant object that was focused on.

\subsection{Evaluation}

These structured and unstructured experiences were tested with a group of four Classics students at Chatsworth House in Derbyshire, UK, as they interpreted two classic Roman statues in the collection. Three members of this group were undergraduate students, and the fourth was a postgraduate student. They were identified as potential users of this type of system, if it were to be deployed in formal or informal learning settings and were recruited directly via University mailing lists.

Due to the lack of an accessible WiFi network or stable mobile data connection on site, a server hosting the system was set up locally using a laptop. This acted as a $\mathrm{WiFi}$ hotspot for the mobile devices to connect to and hosted the activity structures and media content. An additional advantage of this approach was that media transfer was fast and reliable when compared to mobile data; thus, users were not significantly slowed down when uploading video-, audio-, or image-based responses.

In order to gain familiarity with the Android smartphones provided for the trial and their media capture capabilities, the participants were initially invited to collect data from artefacts of their own choice at the site. After this introductory activity, participants were instructed to take part in the structured activity designed for a specific statue group in the collection displaying an older and a younger woman in interaction with each other. Participants worked around each statue at the same time.

This structured activity was then followed by the unstructured activity described above, where the participants were asked to work on a second artefact and collect media, without being given any specific instructions; in this way, the second activity involved again the possibility of submitting images, video/audio recordings and text entries, but did not provide concrete questions; participants were free to follow their own approach to recording their interpretation of the artefact. By using this ordering, the research team intended to observe the extent to which 
participants exposed to the structured activity first (based on the art-historical analysis trajectory employed by experts) would learn and so be able to repeat and apply the learned process of interpretation.

After the session, participants were asked to give feedback on their experiences with the activity and the technology. These data were collected through individual audio-recorded semi-structured interviews. These first prompted participants to relate the positive and negative aspects of the experience. Participants were prompted to give responses with regard to the interface design, the activity design, and the framing and scaffolding provided by the activity. They were also asked to consider if the prototype could be used as an assessment tool, and if so, if they would prefer this as a group activity or an individual task. Finally, they were encouraged to give any other comments.

In order to reflect on the impact of the experience after the event, a 2-h focus group session was conducted in the week following the visit. In this activity, participants and researchers explored the media captured during the visit, with commentary given by participants as to their reasons for capturing particular media, further reflection on how the activity had informed learning and might form a part of formal education, and discussion of ideas for future iterations of the technology (Fig. 2).

\subsection{Findings}

We focus our description of findings on the use of multimedia to capture and represent aspects of interpretation,

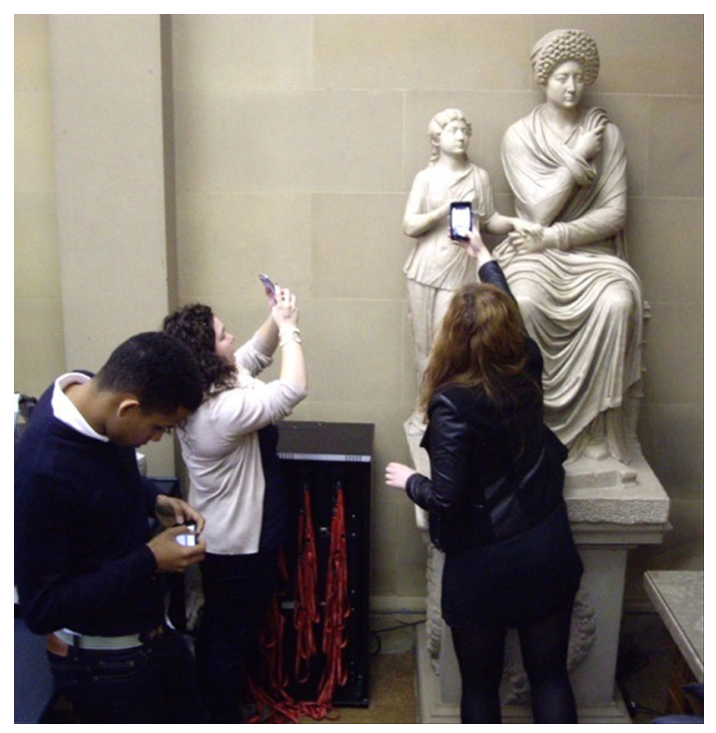

Fig. 2 Participants during the study recording multimedia materials to represent their views of the artefact, in this case a Roman statue group of two female figures and the identifiable ways in which the structures provided impacted on the activities and experiences of the participants.

\subsubsection{Use of multimedia}

Overall, participants spent a greater amount of time than expected carrying out the activity. Although a total of $40 \mathrm{~min}$ for the structured activity was suggested to participants, each spent over an hour on the task. While this amount of engagement was considered positive, we also had concerns that large amounts of time had been spent entering lengthy text responses, which had not been our intention.

The possibility of building a multi-modal interpretation of the artefacts was a common positive in comments from participants, as it helped them to think about the artefact in a more independent and analytical way. Photographs were reported to be the participant's preferred media, since it allowed them 'to go close and get exquisite details' (Participant 2-focus group session). In some cases photographs were used in such a way as to 'to guide the reader, giving a description and an illustrative image' (Participant 2-focus group session). This showed that a factor in the engagement was the notion of producing something that would be later viewed by others.

Taking photographs provided opportunities for creative approaches to representing interpretations within an easyto-use structure. For example, Fig. 3 (top) shows two photographs created by a participant in response to the Systematic Description task. Here, it can be seen that the camera is used to demark sections of the piece. Accompanying text then detailed the features within each of these, for the image on the left, this read:

Woman=Stern face. Full chin. Pressed lips with hollow at corners. Folds either side of nose deep.

...Gaze slightly higher face slightly inclined down.

Massive hair in foamy mass, snail shell curls with interstices drilled. Girl=smaller scale head. Lips pursed in pout...Lighter lidded eyes. Small ears partially covered by hair in extreme waves.

Other participants broke up the systematic description in different ways to this, for example focusing on particular features as show in Fig. 4 (bottom). This provided evidence that while multimedia creation supported structured systematic interrogation and representation of the findings, it also allowed for individual choice and style.

It was observed that audio recordings were not used as expected-as a quick means of capturing responses without resorting to typing. From the participants' interviews, it was highlighted that despite appreciating the capabilities of audio recording to explain the characteristics of the 
Fig. 3 Images taken by participants. P1 (top-left and top-right) passes over the sculpture from top to bottom, $\mathrm{P} 2$ (bottom-left and bottom-right), picks out individual features
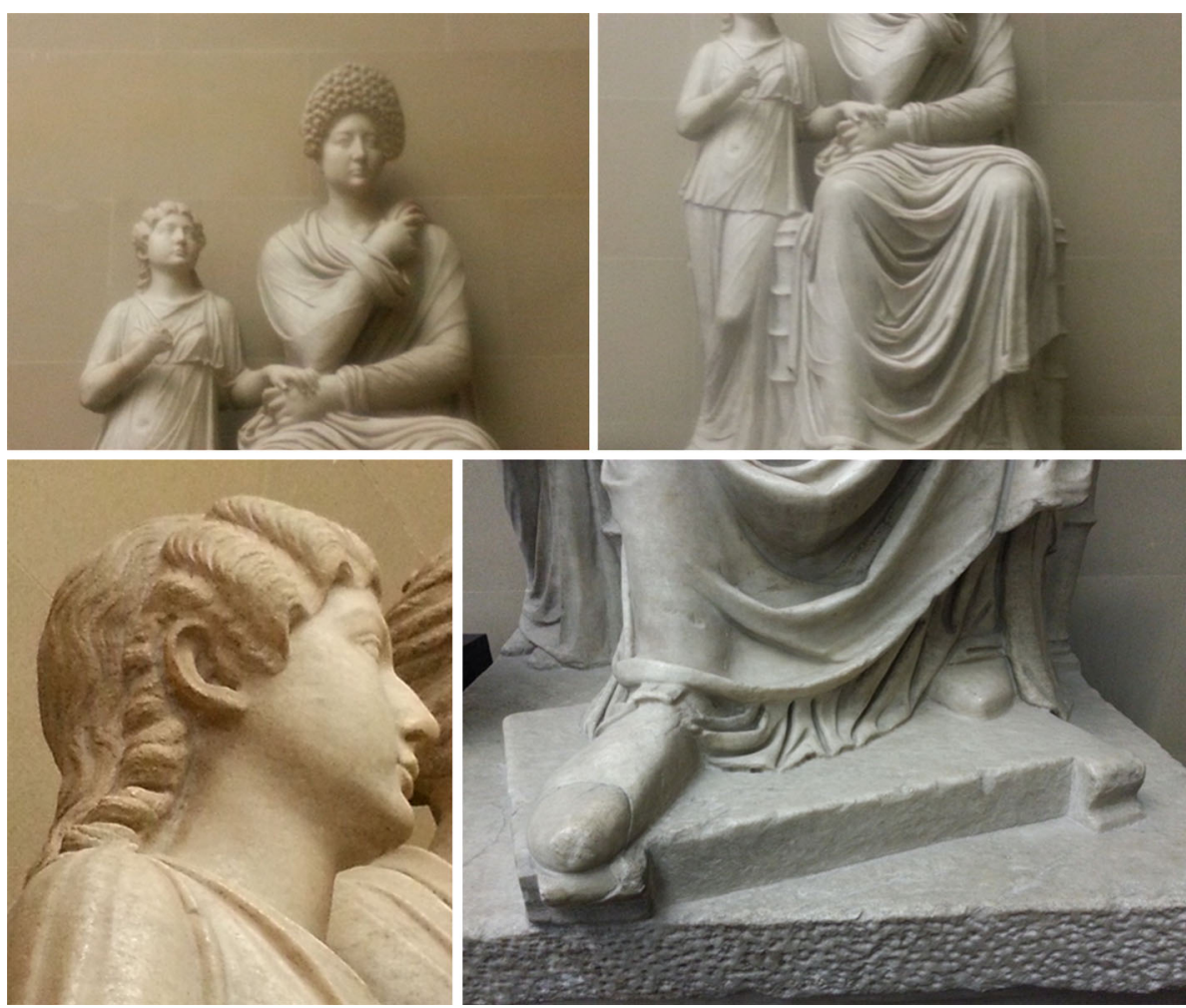

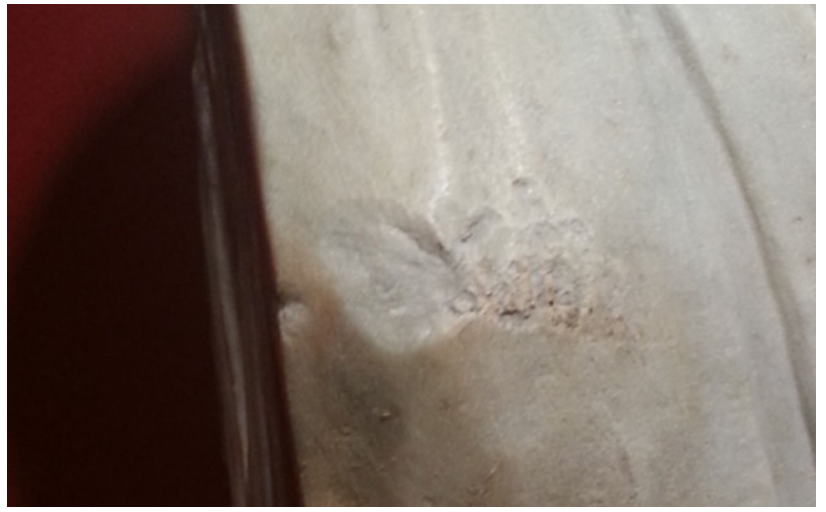

Fig. 4 One of three images submitted by P9 focusing on damaged parts of the artefact

artefact, participants tended to use text entries so that they would not distract other participants from their activities. Similarly, while some participants felt tempted to use video recordings, the fact that there was not much space to move around reduced the opportunities to do so effectively. For example stating that:

'A video would be valuable; but in this case the artefact was it is presented so frontally, so there was not so much space to do that. So even though I did not use it this time, I can think of lot of cases when I would.' Participant 4 - individual audio-recorded interview
Nevertheless, one of the four participants used video recording in most of their responses, affirming that 'I used video for describing the object and text to back it up; video recordings work better for capturing location'. (Participant 2 -focus group session). The same participant stated that using photographs and video recordings to complement text entries could replace time spent on typing descriptions.

\subsubsection{Activity structure}

Regarding the type of activity, all participants agreed that the structured activity was very informative in supporting an appreciation of expert interpretation processes. However, some participants noted that the unstructured activity gave them the potential to demonstrate their knowledge, without limitations or assistance. Reflecting on this, they noted that their level of existing knowledge and confidence were key factors in their enjoyment and benefit of each approach. This is in line with expectations of scaffolding, which suggest that supporting structures are only appropriate in relation to particular levels of knowledge.

In this situation, however, other potential benefits are highlighted in terms of supporting effective learning in the museum context. All of the participants highlighted the importance of following an appropriate process of interpreting an artefact and noted the potential value of the structured activity. e.g.: 
'If you give people an open text box, they would want to go straight forward to the final analysis, whereas taking through those stages, you have to focus on each point and fine details.' (Participant 4 - individual audio-recorded interview)

All the participants agreed that the unstructured activity made them write an essay-like response, but not perhaps a 'good' essay, as they failed to integrate information well. This suggested that the structured activity would help to teach new skills, in order to then write better essays. Their context when conducting this activity - in a museum setting with a mobile device-led them to suggest that they would not conduct any significant research of their own in situ, and also that while they were aware that they should impose some form of structure on their responses, to the unstructured activity, they did not do this well or at all. As such, it is important to consider that the museum context itself can exacerbate difficulties in conducting interpretation-related activities and that structured media creation could augment and overcome these.

Amongst all the tasks, Iconographic Comparison appeared the most popular, as it provided the potential to access other artefacts beyond those present in the museum itself, and use these as context for interpretation. In this way, the system combined the support offered by a procedural structure with additional resources that presented more information to use in interpreting the artefact. This does, however, create a tension with our plans for an artefact-agnostic approach, as comparison activities require objects of relevance to be identified in each case.

When asked to date and interpret the artefact, participants stated that they would have preferred an open text box, since they felt limited with the multiple choice answers presented. More generally, they found these highly structured, closed questions problematic, as they were 'not able to justify answers' (Participant 2-focus group session). Making clarifications such as these is key in interpreting information, particularly where arguments for alternative answers may be made, and closed structures for representing responses do not allow the user to share their opinion effectively. Similar difficulties in producing simple tools to represent these types of information have been raised by other research [4].

One of the participants noted that he would have liked to be aware of how far in the process he was during the activity. This was also addressed in the next iteration of the system, as in some cases participants focused too much on an early activity and subsequently rushed though the later stages.

\section{2nd study: designing an experience for informal museum visiting}

Given the perceived potential of this form of technology for learning in visual analysis and interpretation in museums, a second study was designed focused on testing this concept with the general public. This study built on the existing prototype system, integrating suggested improvements. In this case it was intended that the system would support informal learning, as we were also interested to see whether this approach provided an engaging and enjoyable activity for informal visits to a museum space. In evaluating our approach with both formal and informal learning scenarios, we aim to explore whether a technology could be suitable for use in both settings and so support a larger user base including multiple kinds of museum visitor. In addition, opportunities to increase the attention and procedural knowledge of informal learners could provide a pathway for them to take a greater interest in formal education around the subject.

\subsection{Prototype design}

As participants in the initial study commented on both the value of the structured activity while noting that it could be overly restrictive to their expressive abilities, three different types of experience were designed for the second study. Through these we aimed to further explore potential improvements to the approach:

A highly structured activity very similar to the activity carried out during the first study (see Table 1). Improvements identified in the first study were integrated into this deployment such as changing some ambiguous wording for questions and making pages shorter to minimise the need for scrolling

A semi-structured activity was designed following a defined path, but the answers expected from the users were more open. For example, the multiple choice answers were replaced with open text boxes.

An unstructured activity was used in a similar form to the first study. This was an open activity that facilitated the collection of multimedia materials from the artefacts, but did not include specific tasks.

A modification that was implemented in all the cases was to include an overview of the activity at the beginning, such that users had greater awareness of the entire activity, and should therefore have the awareness to pace themselves appropriately.

Since this study was aimed at the general public and was to be conducted with a larger group of participants, the 
research team identified a different setting, with a larger number of artefacts accessible for the public; Nottingham Castle Museum and Galleries. The exhibition The Treasures of Nemi: Finds from the Sanctuary of Diana focused on the artefacts excavated in 1885 in a temple built in the mid sixth century BC near Rome and represented an excellent example of ancient material culture. Working in collaboration with the curator of the exhibition, the research team identified three artefacts to focus the interpretation experience on during the second study: Double Herm, a double-face head depicting a young man and an old man; Fundilia Rufa, a portrait of a woman on top of a draped herm; and Asklepius, a composite sculpture consisting of face and torso.

Each participant carried out two different activities, one more structured than the other. In contrast with the first study, participants were allocated to different series of activities. Three paths were designed, and each of the participants took part in two types of activities in sequence as follows:

(a) Unstructured activity + Semi structured activity (5 people in total experienced this path)

(b) Highly structured activity + Unstructured activity (4 people in total accessed this path)

(c) Semi structured activity + Unstructured activity (5 people in total carried out this path)

\subsection{Evaluation}

The second study took place at the Nottingham Castle Museums and Galleries. Over 2 days, the research team received participants in three sessions per day at the exhibition space. Each of the sessions was planned to last an hour and including an introduction on the technology and two activities.

A total of 15 participants were recruited, but one withdrew from the study, leaving 14 participants, none of whom had a formal knowledge in classics or the analysis of artefacts. As in the first study, since there was no reliable wireless network at this location, participants accessed the activity from the mobile devices provided through a laptop acting as a Wi-Fi hotspot and hosting the server for the interpretation system.

Before carrying out the task, participants were invited to walk around the gallery space and familiarise themselves with the exhibition. Participants were then provided with a mobile device and invited to proceed with their selected path. Participants were advised to spend around $20 \mathrm{~min}$ on each activity. Afterwards, participants were given an interpretation of the artefacts written by an expert on the subject, to compare with their answers. They then provided feedback on their experiences with both the technology and the activity, through a survey and short discussion.

\subsection{Findings}

As this second trial had a greater number of participants and therefore offered a larger amount of media to analyse. Generally participants enjoyed the experience of using the mobile platform: Twelve of the fourteen participants who completed the study reporting a positive experience, with the remaining two feeling frustrated due to technical issues with uploading media that slowed their progress.

\subsubsection{Analysing reflections}

Within each task participants produced a series of reflections about each artefact, using a range of media (text, photos, audio recordings and video). In the first stage of analysing these reflections, all media submitted by each participant was collated, then broken down in elements that describe/discuss a single aspect of the artefact and are meaningful in isolation. Across the media produced by the 14 participants that completed the experience a total of 157 elements were identified, containing a mix of text, photos, audio recordings and video recordings.

\subsubsection{Use of multimedia}

While none of the participants specifically mentioned media capture strategies or preferences, looking at the number of types of media used, and the frequency of using particular media types to capture elements reveals varied approaches to the tasks, as illustrated in Table 1. For example, $\mathrm{P} 1$ preferred to use more than one type of media to describe elements, while P5 only ever used a single media type to describe a given element (Table 2).

In general text and photos are by far the most popular media for communication of elements. Nearly a quarter of the elements were described with the use of more than one type of media, showing there is some value in allowing the use of multiple media types within tasks. P8 was unusual in having a strong preference for using audio in comparison with other media. Video was used sparsely, but overall, the findings suggest high individual variability in the styles of media use, within the structures given.

\subsubsection{Thematic analysis: types of engagement with the artefact}

In order to explore how the relationships between the capture of elements and engagement with the artefacts, a thematic analysis was performed [2] to categorise elements in terms of interaction with and consideration of the artefact. A total of four themes were identified through this analysis. 
Table 2 Breakdown of use of media to describe artefact elements by participant

\begin{tabular}{lrrlllc}
\hline ID & Text & Photo & Audio & Video & Multimedia & Total elements \\
\hline P1 & 3 & 5 & 4 & 0 & 5 & 7 \\
P2 & 10 & 8 & 4 & 1 & 5 & 18 \\
P3 & 4 & 4 & 2 & 0 & 2 & 8 \\
P4 & 5 & 2 & 0 & 0 & 1 & 6 \\
P5 & 5 & 9 & 0 & 0 & 0 & 14 \\
P6 & 9 & 9 & 7 & 0 & 6 & 18 \\
P8 & 2 & 1 & 7 & 1 & 1 & 10 \\
P9 & 10 & 7 & 0 & 2 & 4 & 15 \\
P10 & 5 & 4 & 1 & 0 & 2 & 8 \\
P11 & 6 & 3 & 1 & 0 & 2 & 10 \\
P12 & 10 & 11 & 2 & 1 & 5 & 19 \\
P13 & 1 & 9 & 1 & 1 & 1 & 10 \\
P14 & 9 & 6 & 1 & 1 & 4 & 13 \\
P15 & 0 & 1 & 0 & 0 & 0 & 1 \\
Tot. & 79 & 78 & 30 & 7 & 38 & 157 \\
\hline
\end{tabular}

Duplication (18 elements) These elements involved simple re-creation of the information provided by the museum and represent a low level of engagement. For example, recording:

'Hemicycle room where the sculptures of the Emperor Tiberius and his sons Germanicus and Drusus were also found.' P5 text, an exact copy of the text on the plinth beside the artefact.

Description (77 elements) Description elements involve a higher level of engagement in order to identify or pick out interesting aspects of the artefact and describe them in some detail. This theme is common when participants used only photographs to focus on an aspect of the artefact without providing text- or audio-based reflections. This type of engagement was the most common and evident across all types of elements.

Personal reflection (5 elements) In a few elements participants enhance descriptions with reflections about how the artefact relates to their personal experiences. While this theme was rare, it was important as it shows how participants could draw on other experiences in order to complete the activities. For example:

'Personally I feel put off by the brashness and haughtiness in the work. There doesn't seem to be a deeper layer to its rather unsophisticated celebration of masculine divinity and the structural damage incurred has left an impoverished image' $\mathbf{P} 3$ text

Interpretation (57 elements) It was relatively common for participants to interpret elements and make implications about the artefact that go beyond the obvious or highly visible aspects of the artefact. These elements are evidence of high level of engagement as participates are internalising information about the artefact in order to extrapolate something about it. For example:

'The sculpture is in good state, clearly showing the different parts it is made of. However it misses some parts such as the arms which can be inferred by the presence of iron fixings at the shoulders.' P2, text.

\subsubsection{Thematic analysis: focal elements}

In order to explore the types of elements identified by participants, a second thematic analysis was performed, this time focusing on what aspect of the object(s) were being considered by the participant. A total of eight themes were identified through this analysis.

Comparison between artefacts (16 elements) These elements describe the artefact in relation to other historical artefacts. These types of element are only present in the semi- and high-structured activities when comparison with other artefacts is explicitly elicited. This suggests making these sorts of comparisons is not something a non-expect will consider unless prompted. For example:

'Similarities appear in the clothing with a long tunic and a mantle worn over it in both artefacts.' - P11, text

Overview (30 elements) Discussing the artefact as a whole, this type of element was one of only two types in which interpretations were the most common form of engagement. For example:

'Seems quite forlorn or wistful' $\mathbf{P 9}$ text.

Components (42 elements) Looking at parts of the artefact in isolation, at times in order to gain insight into the nature of the artefact as a whole. This was by far the most common type of element described as most participants described multiple components for each task they performed.

Construction methods (16 elements) A few participants described the process and tools used to produce the artefact as well as the materials used, but generally only when specifically prompted by the content of the highly structured task. For example:

'Plaster cast made at the end of 1800 of an original of stone.' - $\mathbf{P 2}$ text

'Plaster and originally seated on a stone plinth.' P5 audio recording accompanied by identical text. 
Current location (10 elements) These elements are explorations into the current physical location of the artefact and tended to involve description. For example P14 recorded a short video pointing away from the artefact and panning 360 degrees around the room.

Damage/condition (22 elements) Identifying any damage to the artefact or describing its current condition in more general terms. The interpretation type of engagement as defined above was relatively common in these elements, as participants tended to theorise about the reasons for the current condition of the artefact. For example see Fig. 5.

Discovery location (10 elements) Where and how the artefact was discovered (in modern times), these elements are almost all based on the information provided by the museum on wall plaques. This type of element was the most commonly liked with duplication with 9 of the 10 elements simple copying information provided by the museum. This suggests that more might be done to support personal interpretation around the discovery location, to go beyond these simple duplication strategies.

'Hemicycle room where the sculptures of the Emperor Tiberius and his sons Germanicus and Drusus were also found.' P5 text, accompanied by a photo of a wall plague with identical text.

Provenance (11 elements) When and why the artefact was created. While this could be seen as a sub theme of overview, the nature of these elements was qualitatively different with more obvious narrative aspects. For example:

'It might have been a dedication to the temple of a freed slave who wanted to honour his previous mistress who freed him and was at the same time the priestess at the temple in whose theatre he performed as an actor.' P11 text.

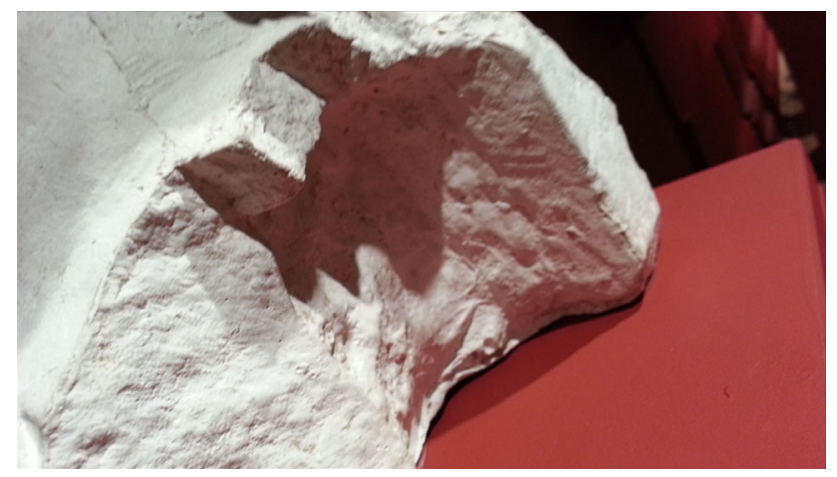

Fig. 5 Image captured from the rear of 'Double Herm' by P12
The relative frequency of elements in these themes suggests that participants are more inclined to talk about the physically presence of the artefact rather than more conceptual aspects. The more common themes of overview, components and damage/condition elements generally focus the physicality of the artefacts rather than the more reflective or implied concepts such as provenance, discovery location or personal reflection.

\subsubsection{Individual experiences}

Next we explore how the data captured provided a picture of the experience of individual participants. Here, we describe the experiences of three participants in detail in order to bring to light contrasting themes.

Example 1: The confidence to interpret P5 is a postdoctoral researcher in a physics department with minimal interest in art and antiquity. She was assigned to 'path c', a semi-structured interpretation of Asklepius followed by an unstructured interpretation of the Double Herm.

It was clear that P5 was not initially comfortable with the first interpretation task, as on several occasions she asked the researcher if she was doing the correct thing, and repeatedly cited her lack of experience in this area. She used a combination of photos and text to capture seven elements, often relying on replication of the text provided on the plaque next to the artefact.

Despite the unstructured nature of their second interpretation task, the elements recorded by P5 show clearly that they were following the structure presented in the first tasks, by first describing the various components of the artefact before offering some well thought-out interpretations, such as stating that:

'The rasp marks on the left side of the chest suggest that another material, or different coloured marble, would have covered this area of the shoulder.' P5 text, Double Herm.

Discussions with P5 after the study revealed that while they found task 1 quite difficult, given their lack of knowledge, being exposed to the process used by professionals gave them the confidence to make interpretations of their own during task two. Her feedback focused on the value of this type of technology to empower members of the public, stating that:

'It breaks a lot of barriers allowing communication and interaction.' Feedback from P5

At the same time, P5 also suggested that there was a steep learning curve associated with the semi-structured task that they had encountered first. 
Example 2: Noticing the details $\mathrm{P} 12$ is a Software Developer with a passing interest in History. During the study briefing he mentions that he often visits museums, but does not have great understanding of the artefacts he sees. He was assigned to 'path b' a highly structured then unstructured activity looking at Fundilia Rufa and Double Herm in turn.

During task one he described a total of 14 elements providing insights across a range of themes including Construction Methods, Provenance, Components and Overview. P12 got very close to the artefact throughout the activity, capturing video and still close-up images of the artefact which he accompanied with text descriptions. For example, when describing Damage/Condition he squeezed behind the plinth on which the exhibit was placed in order to capture images of some signs of damage facing the wall and generally exploring the aspects of the artefact that were hidden from the casual observer, see Fig. 5.

Again during task two, without the structure of microtasks to guide him, he went out of his way to investigate the details of the artefact and went on to draw conclusions based on what he finds. Similarly to P5, he applies the processes he was exposed to during a more structured activity to guide his exploration of the second artefact.

'I can now see that there are flat plains where this (the hair) would have joined.' P12, text.

P12's feedback highlighted an appreciation for the detailed guidance provided in the highly structured task, which led him to explore the artefact in more depth than he otherwise would have. He enjoyed having specific questions to go out and answer and then continued to explore these details with the second artefact unguided, stating that:

'It was good being led through the analysis of the artefacts in a structured way. It made me think about them in more detail. I noticed details that I wouldn't have seen otherwise'. Feedback from P12.

Example 3: The importance of effective scaffolding P15 was one of only two participants who took part in the activity on their own, as while it was an individually focused experience, most participants were physically sharing the space with one to three others who took part simultaneously. She is a Lecturer in History and had no previous experience of classics or the interpretation of historical artefacts. She was assigned to 'path a' consisting of an unstructured experience with Rufa followed by a semi-structured activity with Asklepious.

This participant really struggled with the unstructured activity, ultimately only providing overview images of the artefact without any explanatory text, video, or audio.
During the task she moved around the exhibit looking at plaques describing Rufa and other artefacts. During her second task she did not record any elements, reporting that she did not know the answers to the questions and lacked the confidence to offer up her own interpretations. She explained that:

'I expected to be given some information about the artefacts in addition to what was on the wall' feedback from P15.

Discussions with the participant during and after the experience revealed two key factors that led to her generally negative experience. Firstly, as she performed the task on her own and during a quiet period, she felt unsupported without any peers with whom to relate or take a lead from. As noted by other researchers [7, 14] this feedback highlights the value of peer support when engaging with mobile learning technologies.

Secondly, the fact that she experienced the unstructured activity first meant that she felt unsupported by the technology, as it simply presented open questions with minimal advice on how to go about answering them. Following on from this she did not engaged with the second task in any meaningful way, reading some of the information but not capturing anything. She noted that:

'I felt the questions were putting me on the spot and I

reacted by brain-blanking.' Feedback from P15

This highlights the impact of ordering, as well as of the nature of the scaffolding. Here, it could be argued that an initial lack of support caused the participant to lose confidence and ultimately disengage from the activity.

These three examples highlight both the potential of the prototype system and potential pitfalls to avoid when deploying it 'in the wild'. Drawing together the findings from both studies, the next section will explore implications of this work for wider practice for deploying personal and ubiquitous computing technologies in museum settings.

\section{Discussion}

Our findings highlight that this type of approach could be used to mediate structures for interpretation that are devised by experts, and delivered via technology and the user generation of media. By exploring this through both formal and informal learning, we have managed to unpack some of the issues around varied levels of expertise, confidence, and motivation to engage with this process. In relation to this, our discussion focuses on drawing implications from the results of structuring of the activities, and 
the use of multiple media types in engaging users in these activities.

\subsection{Structure, scaffolding and confidence}

The scaffolding provided by the structured activities was appreciated by many of the participants and particularly those with less prior knowledge and experience. This observation is in line with findings from formal education, where scripting of activities by technology is often recommended [7, 24]. It was not clear at the beginning of the project how this could work within the context of museum visiting outside of formal education, given the considerable differences between the two contexts [6]. As an example the impact of this approach, $30 \mathrm{~s}$ of 'dwell time' is considered to be the normal expectation of visitor attention per artefact [13]. In both our studies, we found that participants engaged with each artefact for far longer, usually over $30 \mathrm{~min}$. This behaviour suggests that structures taken from formal settings can be successfully used to encourage active engagement with museums objects, supporting transitions from capture to more systematic descriptions and personal interpretations.

While our focus was on providing procedures for museum visitors to follow, additional information and resources were effective in supporting comparisons with relevant artefacts from collections outside of the museum. This expands the process of interpretation beyond the pieces available to view and provides the visitor with information that the expert would be aware of, alongside the scaffolding of an interpretation process. In this sense, we augment both the procedural and factual knowledge of the user. While procedural knowledge was the greater focus of our interest, our findings suggest that intertwining this will relevant factual resources is beneficial. However, this produces a tension in terms of an artefact-agnostic approach to structuring media creation, as relevant material for an object must be identified to form part of the system.

From our studies, it would appear that there were both cognitive and affective benefits of this scaffolding. In cognitive terms, interpretation skills such as attending to perceptually subtle details that provide important clues to interpretation would have otherwise been missed. In affective terms, it was clear that participants became more confident to make an interpretation, rather than just look superficially or state an obvious interpretation. At the same time, and in line with the accepted approaches to scaffolding learning in formal education, our findings suggest that it is important to support the fading these structures when the user has built the knowledge and confidence to no longer find it useful. The affective support provided by the scaffolding was also noticeable, and this benefit is in line with the original account of scaffolding theory [26], which often only been addressed cognitively in the subsequent literature.

Particularly in formal education, there is a desire to demonstrate ability that requires that structures may be removed. But conversely, the mobile-in-museum context is not ideal for building coherent arguments, and media creation structured around a generic artefact interpretation process could still enhance the ability of the capable user to produce useful, well-organised outcomes from their visits.

\subsection{Capturing media as engagement with interpretation}

Capturing media during museum visits is a common, yet somewhat controversial activity [1]. Smartphones and other mobile devices offer ever more sophisticated possibilities for this [3]. As such, our aim was to harness various forms of digital media as a form of active engagement with the objects, beyond passive viewing. In designing our structured experiences, smartphone functionality allowed four types of data capture-text, audio, images, and video- to be suggested as the means of producing a response.

Our findings suggest that there is greater comfort with recording images and text, and a particular concern with recording voice into audio and video, due to the potential for interruption to others in the space, and a lack of norms of performing this type of media capture. However, there was evidence of the value of video and audio where it was used, and further design work could look to integrate this better, both in terms of the technology, and in the design of the museum exhibit themselves. For example, certain spaces in an exhibit could be identified in which recording voice was explicitly welcomed by the museum.

The creation of a photograph or other media offers potential for the individual to choose their own method and style [15], while also working within the suggested structure as discussed in the previous section. For example, taking different approaches to 'systematic description' by partitioning up the artefact as was seen fit. Producing media is often a natural response to visiting experiences. As suggested by [5], the structured, yet personal, multimedia outcomes of these interpretation processes could become valuable mementos, which would act as a further motivation and framing to take part in these activities.

The thematic analysis of the outcomes of the second trial presented above provides a first conceptual basis for designing further activities using this approach, and for exploring the aims that might be achieved. For example, we can argue that the observed duplication of information from official sources such as information panels is indicative of a failure to provoke or support individual interpretation. Designers may also consider that a positive 
outcome would include a balance of overview elements showing interpretation and components that highlight that the user is 'noticing the details'.

\section{Conclusions}

In this paper, we have presented a novel approach to utilising expert procedural knowledge in the interpretation of artefacts in museums. We find evidence that structured capture of media has potential to engage, increase understanding, and promote confidence amongst informal visitors to museums, and also has potential to support formal learning activities. One issue to consider in designing such experiences is the combination of abstract interpretation structures, which can be applied across artefacts, and the provision of informational content that is relevant to a particular artefact.

Our exploration of this area has suggested a number of avenues for further research and development. Firstly, there is potential for collecting and sharing these interpretations as a way of crowdsourcing museum visiting experiences. Structures for interpretation could also act as structures that make data usable in a variety of ways, from collecting interpretations and media to assessments of how the public view a particular artefact. The process and outcomes may have value as part of opening up authority and supporting personal interpretation in the museum, as part of formal education, informal learning, and in crowdsourcing structured interpretations of artefacts.

In formal learning, structuring interpretation activities through a procedure that follows a professional vision could provide a way that students could visit sites of interest with some of the benefits of expert supervision. These could also lead to 'embodied assessments' that test the student's abilities in situ, rather than requiring them to complete written assignments and exams that are detached from the subjects and contexts of their work. In informal museum visits, the potential to increase confidence in the public's ability to interpret artefacts could provide a means to opening up of authority in the museum, a key trend as these institutions aim to increase their audience and form new types of relationship with them.

Finally, as wearable and mobile technologies augment our senses and make both providing guidance in situ, and the capture of media from our surroundings even more accessible, the capacity to structure our interpretation of the world around us becomes ever more central to HCI. Allowing users to follow expert procedures while still having flexibility and autonomy within these structures appears as a very attractive way to augment experience.
Acknowledgments This work was funded by the RCUK Horizon Digital Economy Research Hub Grant, EP/G065802/1.

Open Access This article is distributed under the terms of the Creative Commons Attribution 4.0 International License (http://crea tivecommons.org/licenses/by/4.0/), which permits unrestricted use, distribution, and reproduction in any medium, provided you give appropriate credit to the original author(s) and the source, provide a link to the Creative Commons license, and indicate if changes were made.

\section{References}

1. Bitgood S, Cleghorn A (1994) Memory of objects, labels, and other sensory impressions from a museum visit. Visit Behav 9(2):11-12

2. Braun V, Clarke V (2006) Using thematic analysis in psychology. Qual Res Psychol 3(2):77-101

3. Clough G, Jones AC, McAndrew P, Scanlon E (2008) Informal learning with PDAs and smartphones. J Comput Assist Learn 24(5):359-371

4. Coughlan T, Adams A, Rogers Y, Davies S (artefact 1) Enabling live dialogic and collaborative learning between field and indoor contexts. In: Proceedings of the 25th BCS conference on humancomputer interaction. ACM, pp 88-98

5. Durrant A, Rowland D, Kirk DS, Benford S, Fischer JE, McAuley D (2011) Automics: souvenir generating photoware for theme parks. In: Proceedings of ACM conference on human factors in computing systems (CHI'11). ACM, pp 1767-1776

6. Falk JH, Dierking LD (2000) Learning from museums: visitor experiences and the making of meaning. Altamira Press, Lanham, MD, USA

7. Fischer F, Kollar I, Mandl H, Haake JM (2007) Scripting computer-supported collaborative learning: cognitive, computational and educational perspectives, vol 6. Springer, Berlin

8. Fosh L, Benford S, Reeves S, Koleva B (2014) Gifting personal interpretations in galleries. In: Proceedings of ACM conference on human factors in computing systems (CHI'14). ACM, pp 625-634

9. Gaver W, Beaver J, Benford S (2003) Ambiguity as a resource for design. In: Proceedings of CHI'03. ACM, pp 233-240

10. Goodwin C (1994) Professional vision. Am Anthropol 96(3):606-633

11. Henkel LA (2014) Point-and-shoot memories the influence of taking photos on memory for a museum tour. Psychol Sci 25(2):396-402

12. Hofstein A, Rosenfeld S (1996) Bridging the gap between formal and informal science learning. Stud Sci Educ 28:87-112

13. Hsi S, Fait H (2005) RFID enhances visitors' museum experience at the Exploratorium. Commun ACM 48(9):60-65

14. Lewis S, Pea R, Rosen J (2010) Collaboration with mobile media-shifting from 'participation' to co-creation'. In: Proceedings of the sixth international IEEE conference on Wireless, Mobile and Ubiquitous Technologies in Education (WMUTE), pp $112-116$

15. Mayer RE, Massa LJ (2003) Three facets of visual and verbal learners: cognitive ability, cognitive style, and learning preference. J Educ Psychol 95(4):833

16. Panofsky E (2012) On the problem of describing and interpreting works of the visual arts. Translated by Jaś Elsner and Katharina Lorenz. Critical Inquiry, The University of Chicago Press, 38 (3): $487-462$ 
17. Pedaste M, de Jong T, Sarapuu T, Piksööt J, van Joolingen WR, Giemza A (2013) Investigating ecosystems as a blended learning experience. Science 340(6140):1537-1538

18. Phillips LB (2013) The temple and the bazaar: Wikipedia as a platform for open authority in museums. Curator Mus J 56(2):219-235

19. Prince M (2004) Does active learning work? A review of the research. J Eng Educ 93(3):223-231

20. Quintana C, Reiser BJ, Davis EA, Krajcik J, Fretz E, Duncan RG et al (2004) A scaffolding design framework for software to support science inquiry. Scaffolding Spec Issue J Learn Sci 13(3):337-386

21. Rogers Y, Connelly K, Hazelwood W, Tedesco L (2010) Enhancing learning: a study of how mobile devices can facilitate sensemaking. Pers Ubiquitous Comput 14:111-124

22. Rowland D, Porter D, Gibson M, Walker K, Underwood J et al (2010) Sequential art for science and CHI. In: Extended abstracts of ACM conference on human factors in computing systems (CHI'10). ACM, pp 2651-2660
23. Sengers P, Gaver B (2006) Staying open to interpretation: engaging multiple meanings in design and evaluation. In: Proceedings of the 6th conference on designing interactive systems (DIS '06). ACM, pp 99-108

24. Sharples M, Scanlon E, Ainsworth S, Anastopoulou S, Collins T, Crook C, Jones A, Kerawalla L, Littleton K, Mulholland P, O'Malley C (2014) Personal inquiry: orchestrating science investigations within and beyond the classroom. J Learn Sci. doi:10.1080/10508406.2014.944642

25. Trofanenko B (2006) Interrupting the gaze: on reconsidering authority in the museum. J Curric Stud 38(1):49-65

26. Wood D, Bruner J, Ross G (1976) The role of tutoring in problem solving. J Child Psychol Psychiatry 17:89-100

27. Zhao R, Orey M (1999) Implementing the scaffolding strategy with the computer. In annual meeting of the Association for Educational Communications and Technology, Houston 